\title{
Neuroimaging measures of iron and gliosis explain memory performance in aging
}

\author{
Anu Venkatesh $^{1}$ @ | Ana M. Daugherty ${ }^{2}$ | Ilana J. Bennett ${ }^{1,3}$
}

${ }^{1}$ Department of Neuroscience, University of California Riverside, Riverside, California

${ }^{2}$ Department of Psychology, Wayne State University, Detroit, Michigan

${ }^{3}$ Department of Psychology, University of California Riverside, Riverside, California

\section{Correspondence}

Anu Venkatesh, Department of Neuroscience, University of California, Riverside, 900 University Avenue, Riverside, CA 92521-0426.

Email: avenk003@ucr.edu

Funding information

National Institute on Aging, Grant/Award

Number: R21 AG054804

\begin{abstract}
Evidence from animal and histological studies has indicated that accumulation of iron in the brain results in reactive gliosis that contributes to cognitive deficits. The current study extends these findings to human cognitive aging and suggests that magnetic resonance imaging (MRI) techniques like quantitative relaxometry can be used to study iron and its effects in vivo. The effects of iron on microstructure and memory performance were examined using a combination of quantitative relaxometry and multicompartment diffusion imaging in 35 young (21.06 \pm 2.18 years) and 28 older (72.58 \pm 6.47 years) adults, who also completed a memory task. Replicating past work, results revealed age-related increases in iron content (R2*) and diffusion, and decreases in memory performance. Independent of age group, iron content was significantly related to restricted (intracellular) diffusion in regions with low-moderate iron (hippocampus, caudate) and to all diffusion metrics in regions with moderatehigh iron (putamen, globus pallidus). This pattern is consistent with different stages of iron-related gliosis, ranging from astrogliosis that may influence intracellular diffusion to microglial proliferation and increased vascular permeability that may influence all sources of diffusion. Further, hippocampal restricted diffusion was significantly related to memory performance, with a third of this effect related to iron content; consistent with the hypothesis that higher iron-related astrogliosis in the hippocampus is associated with poorer memory performance. These results demonstrate the sensitivity of MRI to iron-related gliosis and extend our understanding of its impact on cognition by showing that this relationship also explains individual differences in memory performance.
\end{abstract}

\section{KEYWORDS}

aging, diffusion imaging, gliosis, iron, memory, microstructure

\section{1 | INTRODUCTION}

While the neurobiological basis of neurodegeneration and memory decline are multi-faceted, iron accumulation and gliosis within gray matter are recognized here as two important contributors. Rather than being independent processes, however, evidence suggests that age-related accumulation of intracellular unbound, non-heme iron (Hallgren \& Sourander, 1958; Mackenzie, Iwasaki, \& Tsuji, 2008; Zecca, Youdim, Riederer, Connor, \& Crichton, 2004) can promote activation and proliferation of glia (gliosis; Beach, Walker, \& 
McGeer, 1989). For example, in vitro (Macco et al., 2013; Pelizzoni, Zacchetti, Campanella, Grohovaz, \& Codazzi, 2013) and in vivo (Thomsen et al., 2015; You et al., 2017) studies in animal models have directly linked iron-related inflammation to gliosis and subsequent cognitive decline, specifically, memory decline (Schröder, Figueiredo, \& De Lima, 2013; Weber et al., 2015). The recently proposed Free-Radical-Induced Energetic and Neural Decline in Senescence (FRIENDS; Raz \& Daugherty, 2018) model extends these findings to human cognitive aging and suggests that magnetic resonance imaging (MRI) methods may be sensitive to markers of iron-related gliosis in vivo for the study of cognitive aging. The current study applies this model by characterizing the relationships between iron content, gray matter gliosis and memory performance using a combination of quantitative relaxometry and diffusion MRI in young and older adults who also completed a recall memory task.

Although non-heme iron is essential to neurons and glia for key metabolic functions (e.g., adenosine triphosphate production, neurotransmitter synthesis; Zecca et al., 2004), chronic iron-related oxidative damage can overwhelm endogenous antioxidant defenses (e.g., glutathione; Vilhardt, Haslund-Vinding, Jaquet, \& McBean, 2017) and result in reactive gliosis (Freitas, Ferreira, Trevenzoli, Oliveira, \& Reis, 2017; Zecca et al., 2004). This can occur when large concentrations of intracellular iron outside binding complexes (e.g., ferritin; Connor, Menzies, Martin, \& Mufson, 1990) produce reactive oxygen species (Macco et al., 2013; Mills, Dong, Wang, \& Xu, 2010) that directly stimulate gliosis (Burda \& Sofroniew, 2014). Iron-related oxidative damage and subsequent gliosis drive the cumulative and progressive cognitive declines that are typical in human aging ( $\operatorname{Raz} \&$ Daugherty, 2018). Applying this FRIENDS model, the current study will use individual and age-group differences in gray matter and performance to characterize the nature of the relationship between iron content and gliosis and their joint contributions to memory performance, which has not yet been assessed in humans in vivo.

A well-established MRI approach for measuring iron content is $R_{2}{ }^{*}$ relaxometry (Langkammer et al., 2010). This approach has been used in humans to demonstrate age-related accumulation of iron in the basal ganglia and hippocampus (Daugherty, Haacke, \& Raz, 2015; Ghadery et al., 2015), consistent with human histological studies (Bartzokis et al., 2007; Zecca et al., 2004). Within the basal ganglia, the globus pallidus has the highest iron concentration across the adult lifespan, whereas the putamen and caudate have a moderate concentration in young adulthood and continue to accumulate iron into old age. This contrasts with the hippocampus, which has less iron concentration in young adulthood and modest accumulation with age (Ghadery et al., 2015). These regional and age group differences in iron content may both affect the relationships between iron and gliosis across the basal ganglia and hippocampus.

Gliosis can have several phenotypes within gray matter, including astrocyte swelling (Singh, Trivedi, Devi, Tripathi, \& Khushu, 2016), microglia proliferation (Yi et al., 2019) and increased blood-brain permeability (Simon \& Iliff, 2016). The sensitivity of diffusion imaging to these phenotypes has been validated in animal models of age and acute injury (Badaut et al., 2011; Budde, Janes, Gold, Turtzo, \& Frank, 2011; Debacker, Djemai, Ciobanu, Tsurugizawa, \& Bihan, 2020; Singh et al., 2016; R. A. Weber et al., 2017; Yi et al., 2019; Zhuo et al., 2012) and in vitro human (Grussu et al., 2017) studies using a combination of diffusion imaging and histology. Since diffusion imaging is sensitive to different phenotypes of gliosis, it may be used to characterize how different phenotypes and stages (as outlined in Burda \& Sofroniew, 2014; Sofroniew, 2015) vary by gray matter region, with the expectation that higher iron would be associated with more pronounced gliosis.

Whereas most of the previous studies have used traditional single-tensor diffusion imaging to investigate gray matter gliosis, the current study will use a multicompartment diffusion approach (Neurite Orientation Dispersion and Density Imaging, NODDI; Zhang, Schneider, Wheeler-Kingshott, \& Alexander, 2012). NODDI models diffusion as three separate compartments including restricted (e.g., intracellular), hindered (e.g., extracellular), and free (e.g., cerebral spinal fluid, CSF) diffusion (Fukutomi et al., 2018; Kaden, Kelm, Carson, Does, \& Alexander, 2016; Rae et al., 2017; Zhang et al., 2012), which may allow this approach to be more sensitive to gliosis and its different stages across gray matter regions. When viewed from the perspective of iron-related gliosis, correlations between $R_{2}{ }^{*}$ and NODDI measures across gray matter regions can demonstrate the sensitivity of MRI techniques to regional differences in gliosis staging. For example, in regions with less iron (e.g., hippocampus), early stages of gliosis, including astrocyte swelling (Norenberg, 1994), may be seen as increases in restricted diffusion. In contrast, regions with more iron (e.g., globus pallidus) may also display gliosis associated with microglia proliferation (Yi et al., 2019) and dysregulation of the blood-brain barrier (Andersen, Johnsen, \& Moos, 2014), which can increase hindered and free diffusion. Alternatively, regions with the largest age group differences in iron content (e.g., putamen, caudate) may display the most pronounced gliosis compared regions with smaller age group differences in iron (e.g., hippocampus). In either scenario, the hippocampus is likely to have relatively low levels of gliosis that may nonetheless impact cognition in young and older adults given the critical role of this region in memory performance (Lister \& Barnes, 2009).

The FRIENDS model of cognitive aging predicts a specific, but as yet untested, combined effect of iron-related gliosis on cognition. Previous studies have separately demonstrated that hippocampal iron content (Ghadery et al., 2015; Rodrigue, Daugherty, Haacke, \& Raz, 2013; Schröder, Figueiredo, \& De Lima, 2013) and microstructure (Carlesimo, Cherubini, Caltagirone, \& Spalletta, 2010; Den Heijer et al., 2012; Radhakrishnan, Stark, \& Stark, 2020) relate to recall memory performance. Here, we aim to assess the combined influence of hippocampal iron and diffusion to differences in memory performance using a commonality analysis between $\mathrm{R}_{2}{ }^{*}$ and NODDI measures.

Building on previous animal research and the FRIENDS model of cognitive aging, the current study aimed to characterize relationships among iron $\left(R_{2}{ }^{*}\right)$, microstructure (hindered, restricted, free diffusion) and memory performance (RAVLT delayed) in younger and older adults using a multimodal MRI approach. The primary objectives were to: (1) replicate regional and age group differences in iron content, 
microstructure and memory performance; (2) examine relationships between iron and microstructure in light of the regional and age group differences in iron, and (3) test functional relevance of the iron-microstructure relationship in the hippocampus by examining their contribution to memory performance. Results are expected to show that higher iron concentration (regional difference) and accumulation (age group difference) relates to higher diffusion across the hippocampus and basal ganglia nuclei, with hippocampal iron and microstructure explaining memory performance. Consistent with the FRIENDS model, these results would provide support to the notion that human MRI data can be interpreted using mechanistic hypotheses from the animal research to ultimately better understand cognitive aging.

\section{MATERIALS AND METHODS}

\section{1 | Participants}

Young and older adults were recruited from the University of California, Riverside (UCR) and surrounding neighborhoods. Prior to enrollment, participants were screened over the phone for neurological conditions (e.g., depression, stroke), scanner related contraindications (e.g., claustrophobia, pregnancy), and general cognition using nonvisual portions of the Montreal Cognitive Assessment (MoCA; Nasreddine et al., 2005; Pendlebury et al., 2017). After completing remaining portions of the MoCA in person, all participants exhibited normal cognition with scores $>23(27.3 \pm 1.63)$. One young and two older participants were excluded due to excessive motion in $R_{2}{ }^{*}$ maps. The final sample included 35 young (mean \pm standard deviation $[S D]=21.06 \pm 2.18$ years, median $=20.64$, range $=18-28$, interquartile range $[\mathrm{IQR}]=2.34,24$ female) and 28 older adults (mean $\pm S D=72.58 \pm 6.47$ years, median $=70.16$, range $=65-86$, $\mathrm{IQR}=8.49,13$ female).

All individuals provided informed consent prior to participation in this study. The UCR Institutional Review Board approved the experimental procedures and participants were compensated for their time.

\subsection{Episodic memory test}

The Rey Auditory Verbal Learning Test (RAVLT; Rey, 1941) was administered to assess delayed free recall, measured as the number of items (out of 15) correctly recalled approximately 25 min after completing five immediate free recall trials of the same word list and one immediate free recall trial of a second word list.

\section{3 | MRI scanning protocol}

Imaging data were acquired using a 3T Siemens Prisma MRI (Siemens Healthineers, Malvern, PA) scanner fitted with a 32-channel receiveonly head coil at the UCR Center for Advanced Neuroimaging.
A high-resolution magnetization-prepared rapid gradient-echo (MP-RAGE) image was acquired with the following parameters: echo time $(T E) /$ repetition time $(T R)=2.72 / 2400 \mathrm{~ms}, 208$ axial slices, voxel size $=0.8 \times 0.8 \times 0.8 \mathrm{~mm}$, and GRAPPA acceleration factor $=2$.

Two diffusion-weighted echo-planar imaging (EPI) sequences were acquired with phase-encoding directions of opposite polarity for correction of susceptibility distortions (Andersson, Skare, \& Ashburner, 2003), each with the following parameters: $\mathrm{TE} / \mathrm{TR}=102 / 3500 \mathrm{~ms}$, FOV $=212 \times 182 \mathrm{~mm}$, matrix size of $128 \times 110$, voxel size $=1.7 \times 1.7 \times 1.7 \mathrm{~mm}, 64$ axial slices, and multiband acceleration factor $=4$. For each acquisition, bipolar diffusion encoding gradients $\left(b=1,500\right.$ and $\left.3,000 \mathrm{~s} / \mathrm{mm}^{2}\right)$ were applied in 64 orthogonal directions, with six images having no diffusion weighting ( $b=0 ; 12$ total).

Multi-echo data derived from a 12-echo $3 \mathrm{D}$ gradient recalled echo (GRE) sequence were acquired with the following parameters: $\mathrm{TE} / \Delta \mathrm{TE} / \mathrm{TR}=4 / 3 / 40 \mathrm{~ms}, \quad \mathrm{FOV}=192 \times 224 \mathrm{~mm}$, matrix size $=192 \times 224 \times 96$, slice thickness $=1.7 \mathrm{~mm}$, and GRAPPA acceleration factor $=2$. Magnitude and phase images were saved for later calculation of $R_{2}{ }^{*}$ values.

\section{4 | Region of interest segmentations}

Bilateral hippocampus, caudate, putamen, and globus pallidus were automatically segmented on each participant's MP-RAGE image using FMRIB Software Library's (FSL; Jenkinson, Beckmann, Behrens, Woolrich, \& Smith, 2012) Integrated Registration and Segmentation Tool (FIRST; Patenaude, Smith, Kennedy, \& Jenkinson, 2011), with the flag for three-stage affine registration for hippocampus (as in Venkatesh, Stark, Stark, \& Bennett, 2020). After visual inspection of each region of interest (ROI), caudate segmentations that underestimated the structure were corrected using a flag to increase the number of modes of variation for fitting from the default $(40)$ to the maximum (336; $n=4$ young) and those that were misaligned were corrected using a linear registration between the MP-RAGE and standard brain (Montreal Neurological Institute; MNI) instead of the default subcortical mask ( $n=1$ young). No corrections were needed for the hippocampus, putamen or globus pallidus segmentations.

\section{5 | Iron image processing}

For each participant, iron data were preprocessed using the procedure outlined in Langley et al. (2020). Briefly, $R_{2}{ }^{*}$ values were estimated using a custom script in MATLAB which fit a monoexponential model, $\left(S_{i}=S_{0} \exp \left[-R_{2}{ }^{*} T E\right]\right.$, where $S_{i}$ indicates the signal of a voxel at the $i$ th echo time and $S_{0}$ indicates a fitting constant) to the GRE images.

FSL's FMRIB Linear Image Registration Tool (FLIRT) was used to align the resulting $R_{2}{ }^{*}$ map to the MPRAGE image via the magnitude image from the first echo, using a rigid body transformation (six degrees of freedom, DOF). An affine transformation (12 DOF) with nearest neighbor interpolation was used to align the FIRST segmented 
ROls into iron space using FLIRT and the matrix file from the previous step. Each bilateral iron space-aligned ROI mask was then multiplied by the voxel-wise $R_{2}{ }^{*}$ map before taking the average across voxels and mean $R_{2}{ }^{*}$ was extracted for each participant.

For each bilateral ROI, mean $R_{2}{ }^{*}$ (Iron ${ }_{\text {raw }}$ ) was adjusted for ROI volume using the normalization method from Jack et al. (1989). The FIRST-segmented ROI volumes (Volume ${ }_{\text {indiv }}$ ) were used to calculate adjusted $R_{2}{ }^{*}$ (Iron norm) separately for each participant using the following equation: $\operatorname{Iron}_{\text {norm }}=\operatorname{Iron}_{\text {raw }}-\beta\left(\right.$ Volume $_{\text {indiv }}-$ Volume $\left._{\text {mean }}\right)$. Mean volume (Volume mean $_{\text {) }}$ and slope $(\beta)$ were calculated within the young adults. Volume-adjusted $R_{2}{ }^{*}$ values were used for all analyses.

\subsection{Diffusion data processing}

For each participant, diffusion data were pre-processed using FSL, except that a binary brain mask was created using Analysis of Functional Neuro Images (AFNI; Cox, 1996). After generating a field map using Topup, Eddy was used to correct for distortions due to motion, eddy-currents, and susceptibility (Andersson et al., 2003; Andersson \& Sotiropoulos, 2016).

The NODDI MATLAB toolbox was then used to estimate voxelwise measures of restricted (also known as intracellular volume fraction, ICVF), hindered (also known as orientation dispersion index, ODI) and free (also known as isotropic fraction, fISO) diffusion (http:// mig.cs.ucl.ac.uk/index.php; Zhang et al., 2012). To more accurately model diffusion within gray matter, the intrinsic diffusivity assumption, used to estimate restricted and hindered diffusion, was set to $1.1 \times 10^{-3} \mathrm{~mm}^{2} / \mathrm{s}$ (Fukutomi et al., 2018; Fukutomi et al., 2019; Guerrero et al., 2019).

For each participant, diffusion metrics were extracted separately for each FIRST segmented ROI. A rigid body transformation was used to align the FIRST segmented ROls to diffusion space using FLIRT. For free diffusion, a bilateral diffusion space-aligned ROI mask was multiplied by the voxel-wise free diffusion image before taking the average across voxels. To limit hindered and restricted diffusion metrics to voxels with sufficient tissue content, an inclusion mask was created by thresholding the free diffusion image to voxels with high tissue content (free diffusion $<90 \%$ ). The inclusion mask was then multiplied by each bilateral diffusion space-aligned ROI mask and then by the corresponding voxel-wise diffusion image before taking the average across voxels and hemispheres.

\section{7 | Statistical analyses}

All analyses were conducted using SPSS (Version 24.0; IBM, Armonk, NY). Separate 2 Age Group (young, older) $\times 4$ Region (hippocampus, caudate, putamen, globus pallidus) mixed factorial ANOVAs were conducted for each measure of iron content $\left(R_{2}{ }^{*}\right)$ and diffusion (restricted, hindered, free), with age group as a betweensubjects factor and region as a within-subjects factor. Significant main effects of Regions were followed with post-hoc paired $t$ tests for each pair of regions and significant interactions were followed with post- hoc 2 Age Group $\times 2$ Region mixed factorial ANOVAs for each pair of regions. For these post-hoc comparisons, the significance threshold was adjusted by Bonferroni correction for six comparisons $\left(\alpha^{\prime}=0.008\right)$.

Separate linear regressions were conducted for each diffusion metric (restricted, hindered, free) and each region (hippocampus, caudate, putamen, globus pallidus), with Age Group, $\mathrm{R}_{2}{ }^{*}$, and Age Group $\times \mathrm{R}_{2}{ }^{*}$ as predictor variables. Significant effects were Bonferroni corrected for three comparisons per region $\left(\alpha^{\prime}=0.017\right)$.

To test for neural correlates of the memory measure, it is equally important to consider the correlated effect of iron content and gliosis as well as their unique effects. To determine the shared effect, a commonality analysis was performed (Lindenberger, von Oertzen, Ghisletta, \& Hertzog, 2011). The commonality analysis uses a series of linear regressions to calculate the shared and unique effects of each predictor (iron content and diffusion) and estimates the shared variance of the predictors as a proportion of the total variance explained in memory performance (shared over simple effect; SOS). Large values would indicate high commonality between predictors, which is consistent with the iron-related gliosis model reviewed.

\section{3 | RESULTS}

\section{$3.1 \mid$ Iron content}

An Age Group (young, older) $\times$ Region (hippocampus, caudate, putamen, globus pallidus) repeated measures ANOVA was conducted for $R_{2}{ }^{*}$ (Figure 1). There was a significant effect of Region, $F$ $(3,183)=492.36, p<.001$, with the highest iron content in the globus pallidus $(33.46 \pm 4.72)$, followed by the putamen $(24.27 \pm 4.84)$, caudate $(20.97 \pm 2.57)$, and hippocampus $(16.40 \pm 1.49)$. Post hoc pairwise comparisons revealed that iron content was significantly different between all regions, $p s<.001$.

There were also significant effects of Age Group, $F(1,61)=51.47$, $p<.001$, and Age Group $\times$ Region, $F(3,183)=16.77, p<.001$. Overall, iron content was higher in older adults $(25.81 \pm 0.38)$ compared to young (22.15 \pm 0.34$)$. Post hoc 2 Age Group $\times 2$ Region comparisons revealed that the age group difference was significantly larger in the putamen $(7.29 \pm 0.81)$ compared to the caudate $(3.46 \pm 0.49), F$ $(1,61)=40.10, p<.001$, globus pallidum $(3.06 \pm 1.14), F(1,61)=13.67$, $p<.001$, and hippocampus $(0.83 \pm 0.37), F(1,61)=49.27, p<.001$; and in the caudate compared to the hippocampus, $F(1,61)=49.27$, $p<.001$. The age-related differences in globus pallidus were statistically equivalent to that in the caudate $(p>.20)$ and hippocampus $(p>.05)$.

\section{2 | Microstructure}

Age Group (young, older) $\times$ Region (hippocampus, caudate, putamen, globus pallidus) repeated measures ANOVAs were conducted separately for each diffusion metric. In the event of significant interactions, post-hoc Age Group $\times$ Region ANOVAs for each pair of regions was conducted. 

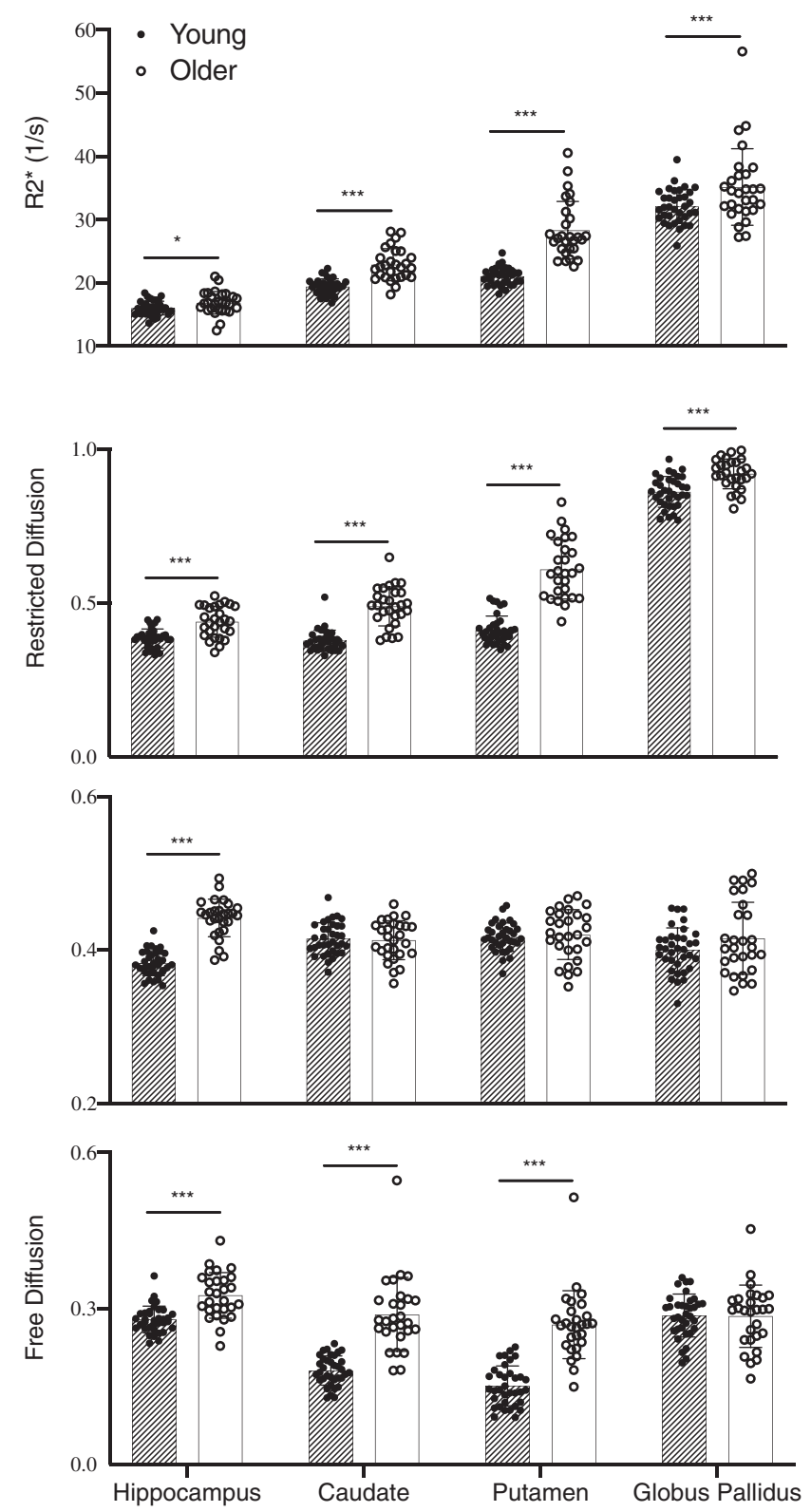

FIGURE 1 Iron content $\left(R_{2}{ }^{*}\right)$ and microstructure (restricted, hindered, free diffusion) are shown separately for young (black circles, stripe bar) and older (open circles, open bar) adults in each region of interest. ${ }^{* * *} p<.001,{ }^{*} p<.05$

\subsection{1 | Restricted diffusion}

There was a significant effect of Region, $F(3,183)=1851.3, p<.001$, with the highest restricted diffusion in the globus pallidus $(0.89$ $\pm 0.06)$, followed by the putamen $(0.50 \pm 0.12)$, caudate $(0.43 \pm 0.07)$, and hippocampus $(0.41 \pm 0.05)$. Post hoc pairwise comparisons revealed that restricted diffusion was significantly different between all regions, $p s<.008$.

There were significant effects of Age Group, $F(1,61)=102.84$, $p<.001$, and Age Group $\times$ Region, $F(3,183)=41.05, p<.001$. Overall, restricted diffusion was higher in older adults $(0.61 \pm 0.01)$ compared to young $(0.51 \pm 0.01)$. Post hoc 2 Age Group $\times 2$ Region comparisons revealed that the age group difference was significantly larger in the putamen $(0.19 \pm 0.02)$ compared to the caudate $(0.11 \pm 0.01), F$ $(1,61)=46.97, p<.001$, globus pallidus $(0.06 \pm 0.01), F(1,61)=78.73$, $p<.001$, and hippocampus $(0.05 \pm 0.01), F(1,61)=65.11, p<.001$; in the caudate compared to the globus pallidus $(0.05 \pm 0.01), F(1,61)$ $=14.95, p<.001$, and hippocampus, $F(1,61)=20.76, p<.01$; and in the globus pallidus compared to the hippocampus, $F(1,61)=0.09, p<.001$.

\subsection{2 | Hindered diffusion}

The effect of Region was not significant, $p>.08$, but there were significant effects of Age Group, $F(1,61)=16.10, p<.001$, and Age Group $\times$ Region, $F(3,183)=21.63, p<.001$. Overall, hindered diffusion was higher in older adults $(0.42 \pm 0.01)$ compared to young $(0.40$ \pm 0.01 ). Post hoc 2 Age Group $\times 2$ Region comparisons revealed that the age group difference was significantly larger in the hippocampus $(0.06 \pm 0.01)$ compared to the caudate, $F(1,61)=74.63, p<.001$, putamen, $F(1,61)=58.46, p<.001$, and globus pallidus, $F$ $(1,61)=19.84, p<.001$. The results did not statistically differ between the remaining regions, $p s>.09$.

\subsection{3 | Free diffusion}

There was a significant effect of Region, $F(3,183)=81.37, p<.001$, with the highest free diffusion in the hippocampus $(0.30 \pm 0.04)$, followed by globus pallidus $(0.29 \pm 0.05)$, caudate $(0.23 \pm 0.07)$ and putamen $(0.20 \pm 0.08)$. Post hoc pairwise comparisons revealed that free diffusion was significantly different between all regions, $p s<.001$, except between globus pallidus and hippocampus, $p>.23$.

There were also significant effects of Age Group, $F(1,61)=58.14$, $p<.001$, and Age Group $\times$ Region, $F(3,183)=34.22, p<.001$. Overall, free diffusion was higher in older $(0.29 \pm 0.01)$ compared to young $(0.22 \pm 0.01)$ adults. Post hoc 2 Age Group $\times 2$ Region comparisons revealed that the age group difference was significantly larger in the hippocampus $(0.05 \pm 0.02)$ compared to the globus pallidus $(0.01$ $\pm 0.01), F(1,61)=9.66, p<.004$, caudate $(0.11 \pm 0.04), F(1,61)=31.74$, $p<.001$, and putamen $(0.12 \pm 0.03), F(1,61)=61.00, p<.001$; and in the globus pallidus compared to the caudate, $F(1,61)=40.81, p<.001$, and putamen, $F(1,61)=58.92, p<.001$. The results did not statistically differ between the remaining regions, $p s>.30$.

\section{3 | Relation between iron and microstructure}

Separate linear regressions for each region tested the relationship between iron content $\left(R_{2}{ }^{*}\right)$ and each diffusion metric, as well as the potential moderating effect of age group (by including Age Group $\times$ $R_{2}{ }^{*}$ as a predictor). Age group was included as a covariate in all models given the previously described age effects. Significant effects were Bonferroni corrected for three comparisons per region $(p<.017$; Figure 2). 

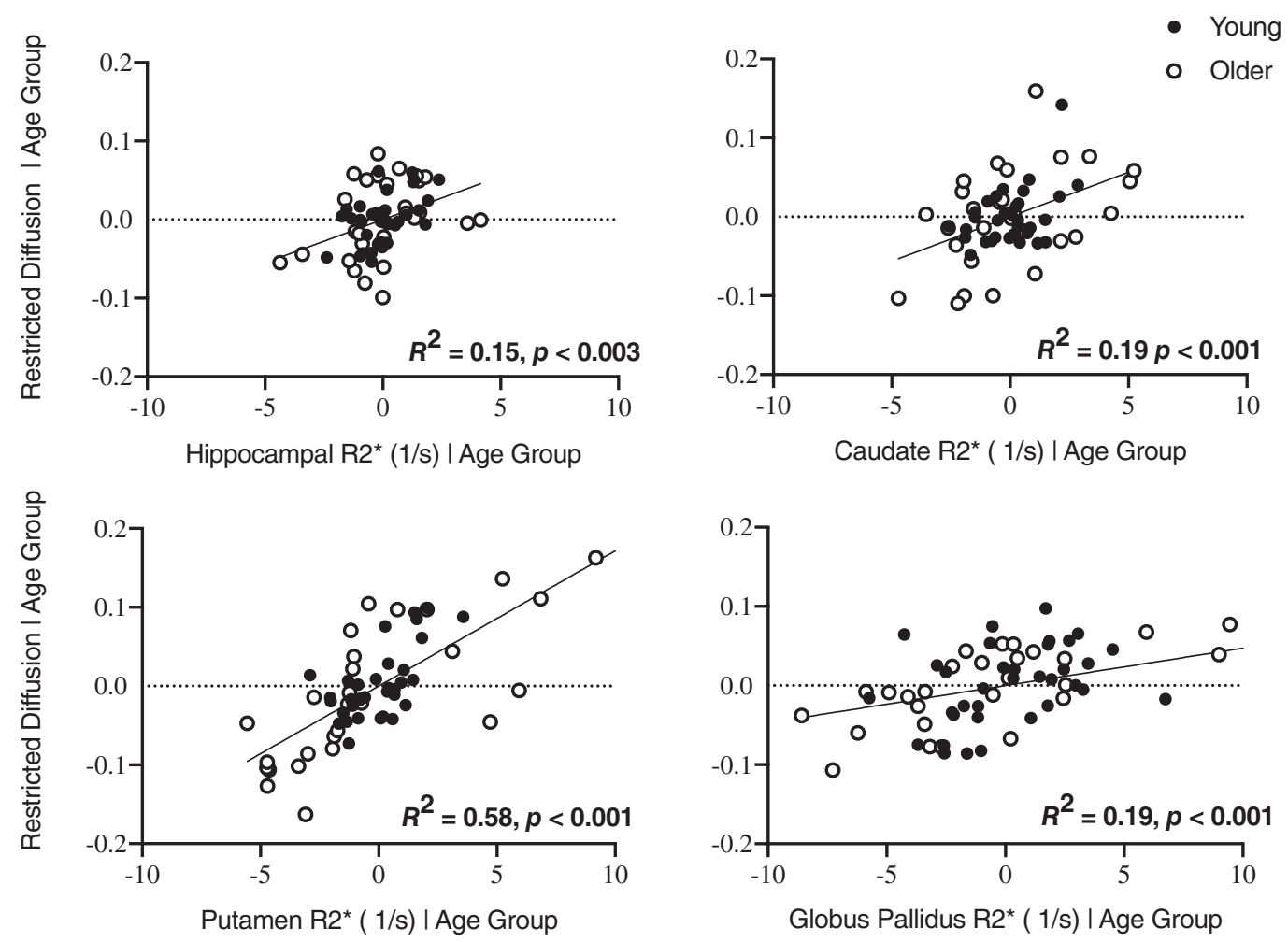

FIGURE 2 Significant associations between iron content $\left(R_{2}{ }^{*}\right)$ and microstructure (restricted diffusion) are shown separately for each region after controlling for age group

For the hippocampus, $\beta=0.34, t(62)=3.31, p<.003$, and caudate, $\beta=0.39, t(62)=3.75, p<.001$, significant positive relationships were observed between $R_{2}{ }^{*}$ and restricted diffusion, but not hindered or free diffusion, $p s>.03$. For the putamen, significant positive relationships were observed between $R_{2}{ }^{*}$ and restricted, $\beta=0.67, t$ $(62)=8.85, p<.001$, hindered, $\beta=0.95, t(62)=6.23, p<.001$, and free, $\beta=0.54, t(62)=4.98, p<.001$, diffusion. For the globus pallidus, significant positive relationships were observed between $R_{2}{ }^{*}$ and restricted, $\beta=0.40, t(62)=3.83, p<.001$, and hindered, $\beta=0.52, t(62)=4.52, p<.001$, diffusion, whereas a significant negative relationship was observed between $R_{2}{ }^{*}$ and free diffusion, $\beta=-0.47, t(62)=-3.83, p<.001$. There was no evidence of age group moderating these relationships in any region, $p s>.10$, indicating that the $R_{2}{ }^{*}$-diffusion relationship was comparable in young and older adults.

\subsection{Contributions of iron and microstructure to memory performance}

An independent sample $t$ test assessed age group differences in RAVLT delayed recall, $t(47)=-4.06, p<.001,95 \% \mathrm{Cl}[-4.90$, $-1.66]$. As expected, older adults $(8.04 \pm 3.59)$ recalled significantly fewer words than young $(11.31 \pm 2.57)$, and performed within $2 \mathrm{SD}$ of the expected normative range for age and education (Lezak, 1995; Messinis et al., 2015).
TAB LE 1 Summary of regression models for the commonality analysis

\begin{tabular}{llrll} 
Model & $\boldsymbol{R}^{2}$ & \multicolumn{1}{c}{$\boldsymbol{F}$} & $\mathbf{d f}$ & $\boldsymbol{p}$ \\
\hline Restricted diffusion & 0.315 & 28.10 & $(1,62)$ & .001 \\
\hline$R_{2}{ }^{*}$ & 0.118 & 8.17 & $(1,62)$ & .006 \\
\hline Total effect & 0.324 & 14.36 & $(2,62)$ & .001 \\
\hline
\end{tabular}

Note: The dependent variable was RAVLT delayed recall.

A commonality analysis quantified the shared variance between hippocampal iron $\left(R_{2}{ }^{*}\right)$ and microstructure (restricted diffusion) in explaining memory performance (delayed free recall). These analyses were limited to the hippocampus due to its known role in memory and to the restricted diffusion metric given its previously described relationship to hippocampal iron. Results revealed that $31.5 \%$ of the variance in delayed recall performance was explained by restricted diffusion alone, $11.8 \%$ by $R_{2}{ }^{*}$ alone, and a total of $32.4 \%$ when both restricted diffusion and $R_{2}{ }^{*}$ were included in the model (see Table 1). From this procedure, of the total variance in RAVLT recall that was explained by diffusion, $34.6 \%$ of the effect was shared with hippocampal $R_{2}{ }^{*}$.

Since the variance in delayed recall performance explained by our metrics of interest may be shared with age, the commonality analysis was repeated after including age group as a covariate 


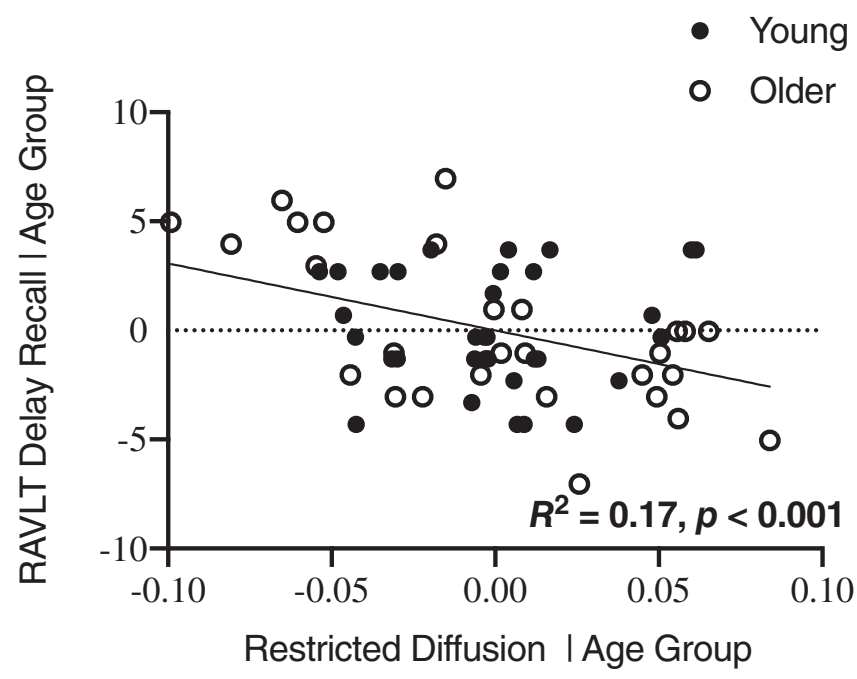

FIGURE 3 Significant association between hippocampal restricted diffusion and RAVLT delayed recall performance, independent of age group

(see Figure 3). In this model, restricted diffusion uniquely explained $12.7 \%$ of variance in delayed recall, $R^{2}=0.13, p<.001$, the unique effect of $R_{2}{ }^{*}$ was $4.8 \%, R^{2}=0.05, p=.05$, and the total effect of both predictors was $13.5 \%$. Therefore, even independent of age group, $31.5 \%$ of the total variance in delayed recall that was explained by hippocampal restricted diffusion was shared with hippocampal $R_{2}{ }^{*}$. Taken together, hippocampus microstructure significantly contributes to memory performance independent of age, and approximately $32 \%$ of its effect is also related to iron content.

\section{DISCUSSION}

The current study tested the relationships between brain iron, gliosis, and memory in humans using a combination of neuroimaging techniques, consistent with the FRIENDS model of cognitive aging. Our results revealed several major findings. First, we replicated wellknown regional and age group differences in iron content $\left(R_{2}{ }^{*}\right)$, tissue microstructure (NODDI) and memory performance (RAVLT delayed recall). Second, we observed relationships between iron and microstructure that were specific to restricted diffusion in the hippocampus and caudate, whereas they were observed in all diffusion measures in the putamen and globus pallidus, consistent with stages of gliosis as a function of regional iron content. Moreover, these iron-microstructure relationships were not moderated by age group, suggesting that the effect of iron on microstructure may be cumulative and progressive across the adult lifespan. Third, restricted diffusion in the hippocampus related to recall memory performance, with a third of this variance shared with iron estimates. These results demonstrate that MRI is sensitive to iron-related gliosis within gray matter, which contributes to individual differences in memory performance.
As expected, we observed age group differences in delayed recall (Bennett, Huffman, \& Stark, 2015), gray matter microstructure (Nazeri et al., 2015; Radhakrishnan et al., 2020; Venkatesh et al., 2020), and iron content (Bartzokis et al., 2007; Ghadery et al., 2015) that replicated previous studies, indicating that these differences are likely part of normal aging processes. Of particular importance given our interest in relationships between iron and diffusion measures, we observed the expected patterns of regional and age group differences in iron. Iron concentration was highest in the globus pallidus and putamen followed by the caudate and hippocampus, whereas age-related iron accumulation was largest in the putamen and caudate followed by the globus pallidus and hippocampus, consistent with previous reports in healthy older adults (Bartzokis et al., 2007; Daugherty \& Raz, 2013; Ghadery et al., 2015). This allowed us to examine the extent to which NODDI metrics are sensitive to various stages of iron-related gliosis across the gray matter regions of interest.

We further observed significant relationships between iron content and microstructure that varied across the hippocampus and basal ganglia nuclei. Within the hippocampus and caudate, relationships between $R_{2}{ }^{*}$ and NODDI metrics were specific to restricted diffusion. Of note, these regions had low to moderate overall iron concentration in spite of having both small (hippocampus) and large (caudate) age group differences in iron accumulation. We interpret this pattern of results as being consistent with an earlier stage of iron-related gliosis (Norenberg, 1994; Pekny \& Nilsson, 2005), in which astrocyte activation and swelling are limited to the intracellular source of diffusion. The positive direction of these effects also supports the notion that higher iron content is accompanied by reactive astrogliosis through oxidative damage, and hence an increase in intracellular sources of diffusion. Proposing astrogliosis as a potential mechanism that influences restricted diffusion extends previous work that has traditionally attributed this diffusion metric to neurite density (Fukutomi et al., 2019; Grussu et al., 2017; Metzler-Baddeley et al., 2019; Radhakrishnan et al., 2020) and provides a parsimonious explanation for previous observations of age-related increases in gray matter restricted diffusion seen by our group (Franco, Petok, Langley, Hu, \& Bennett, 2020; Venkatesh et al., 2020) among others (MetzlerBaddeley et al., 2019; Radhakrishnan et al., 2020).

In contrast, within the putamen and globus pallidus, $R_{2}{ }^{*}$ was related to all three diffusion metrics. These regions had moderate to high overall iron concentration, but both large (putamen) and small (globus pallidus) age group differences in iron accumulation. This pattern of results may indicate later stages of iron-related gliosis in which astrogliosis is coupled with microglia proliferation (Yi et al., 2019) and increased vascular permeability (Elahy et al., 2015) that would influence extracellular and free, not just intracellular, sources of diffusion. Recent evidence supports the notion that hindered diffusion is sensitive to infiltrating microglia, as one study demonstrated that hindered diffusion significantly varied depending on microglia density in mice (Yi et al., 2019). Whereas $R_{2}{ }^{*}$ was only positively related to restricted and hindered diffusion, positive (putamen) and negative (globus pallidus) correlations were seen for free diffusion, which likely reflects low signal to noise ratios in the diffusion signal within the globus pallidus. Taken together, the regional patterns between iron content 
and microstructure observed here appear to reflect an iron concentration-dependent effect on microstructure. As such, our findings are consistent with and extend the iron-gliosis hypothesis in humans by demonstrating that increased iron accumulation in gray matter is accompanied by a glial response that can be detected initially with intracellular (restricted) and then extracellular (hindered, free) diffusion metrics. Further, by finding that the iron-microstructure relationships were comparable between young and older adults across the hippocampus and all basal ganglia nuclei, our results suggest that iron-related gliosis is cumulative and progressive across the lifespan.

Finally, we demonstrated the shared consequence of hippocampal iron-related gliosis on recall memory performance, providing functional relevance to the current findings. Greater hippocampal restricted diffusion explained $31.5 \%$ of the variance in memory performance, $34.6 \%$ of this effect was shared with hippocampal $R_{2}{ }^{*}$. Consistent with our interpretation of a cumulative effect of iron across the lifespan, approximately $31.5 \%$ of shared variance between microstructure and iron estimates remained after statistically controlling for age. These findings extend at least one previous study that observed higher restricted diffusion related to poorer memory performance in younger and older adults (Radhakrishnan et al., 2020) by revealing the extent to which this diffusion-memory relationship is shared with iron. More importantly, these behavioral results provide an important piece of evidence in support of the iron-gliosis hypothesis and FRIENDS model by demonstrating the sensitivity of MRI to iron-related hippocampal astrogliosis as a correlate to memory performance.

In conclusion, the current study revealed key pieces of evidence in support of the iron-gliosis hypothesis in humans. We found significant relationships between iron content and tissue microstructure that systematically varied across subcortical regions (but not age groups) in an iron concentration-dependent manner. This has functional consequences as iron content and tissue microstructure together contribute to recall memory performance, independent of age. Future studies could build on these results by investigating other MRI techniques (e.g., Fluid-Attenuated Inversion Recovery) and cognitive processes (e.g., psychomotor speed) that may influence relationships among iron content, gray matter microstructure and memory performance in aging. Nonetheless, this study represents an important validation and extension of both the animal literature that gave rise to the iron-gliosis hypothesis and the FRIENDS model by demonstrating that MRI is sensitive to individual differences in iron and gliosis, and that their combined effect explains memory performance.

\section{ACKNOWLEDGMENT}

This work was supported by R21 AG054804 (Bennett, IJ).

\section{CONFLICT OF INTEREST}

The authors declare no competing interests.

\section{DATA AVAILABILITY STATEMENT}

The data that support the findings of this study are available from the corresponding author upon reasonable request.

\section{ORCID}

Anu Venkatesh (D) https://orcid.org/0000-0003-3230-8467

\section{REFERENCES}

Andersen, H. H., Johnsen, K. B., \& Moos, T. (2014). Iron deposits in the chronically inflamed central nervous system and contributes to neurodegeneration. Cellular and Molecular Life Sciences, 71, 1607-1622. https://doi.org/10.1007/s00018-013-1509-8t

Andersson, J. L. R., Skare, S., \& Ashburner, J. (2003). How to correct susceptibility distortions in spin-echo echo-planar images: Application to diffusion tensor imaging. Neurolmage, 20(2), 870-888. https://doi.org/ 10.1016/S1053-8119(03)00336-7t

Andersson, J. L. R., \& Sotiropoulos, S. N. (2016). An integrated approach to correction for off-resonance effects and subject movement in diffusion MR imaging. Neurolmage, 125, 1063-1078. https://doi.org/10. 1016/J.NEUROIMAGE.2015.10.019t

Badaut, J., Ashwal, S., Adami, A., Tone, B., Recker, R., Spagnoli, D., ... Obenaus, A. (2011). Brain water mobility decreases after astrocytic aquaporin-4 inhibition using RNA interference. Journal of Cerebral Blood Flow and Metabolism: Official Journal of the International Society of Cerebral Blood Flow and Metabolism, 31(3), 819-831. https://doi. org/10.1038/jcbfm.2010.163t

Bartzokis, G., Tishler, T. A., Lu, P. H., Villablanca, P., Altshuler, L. L., Carter, M., ... Mintz, J. (2007). Brain ferritin iron may influence ageand gender-related risks of neurodegeneration. Neurobiology of Aging, 28(3), 414-423. https://doi.org/10.1016/j.neurobiolaging.2006.02. $005 \mathrm{t}$

Beach, T. G., Walker, R., \& McGeer, E. G. (1989). Patterns of gliosis in Alzheimer's disease and aging cerebrum. Glia, 2(6), 420-436. https:// doi.org/10.1002/glia.440020605

Bennett, I. J., Huffman, D. J., \& Stark, C. E. L. (2015). Limbic tract integrity contributes to pattern separation performance across the lifespan. Cerebral Cortex, 25(9), 2988-2999. https://doi.org/10.1093/cercor/bhu093t

Budde, M. D., Janes, L., Gold, E., Turtzo, L. C., \& Frank, J. A. (2011). The contribution of gliosis to diffusion tensor anisotropy and tractography following traumatic brain injury: Validation in the rat using Fourier analysis of stained tissue sections. Brain, 134(8), 2248-2260. https:// doi.org/10.1093/brain/awr161t

Burda, J. E., \& Sofroniew, M. V. (2014). Reactive gliosis and the multicellular response to CNS damage and disease. Neuron, 81, 229-248. https://doi.org/10.1016/j.neuron.2013.12.034t

Carlesimo, G. A., Cherubini, A., Caltagirone, C., \& Spalletta, G. (2010). Hippocampal mean diffusivity and memory in healthy elderly individuals: A cross-sectional study. Neurology, 74(3), 194-200. https://doi.org/ 10.1212/WNL.0b013e3181cb3e39t

Connor, J. R., Menzies, S. L., Martin, S. M. S., \& Mufson, E. J. (1990). Cellular distribution of transferrin, ferritin, and iron in normal and aged human brains. Journal of Neuroscience Research, 27(4), 595-611. https://doi.org/10.1002/jnr.490270421

Cox, R. W. (1996). AFNI: software for analysis and visualization of functional magnetic resonance neuroimages. Computers and Biomedical Research, 29(3), 162-173. https://doi.org/10.1006/cbmr.1996.0014t

Daugherty, A., \& Raz, N. (2013). Age-related differences in iron content of subcortical nuclei observed in vivo: A meta-analysis. Neurolmage, 70, 113-121. https://doi.org/10.1016/J.NEUROIMAGE.2012.12.040t

Daugherty, A. M., Haacke, X. E. M., \& Raz, N. (2015). Striatal iron content predicts its shrinkage and changes in verbal working memory after two years in healthy adults. Journal of Neuroscience, 35(17), 67316743. https://doi.org/10.1523/JNEUROSCI.4717-14.2015t

Debacker, C., Djemai, B., Ciobanu, L., Tsurugizawa, T., \& Bihan, D. L. (2020). Diffusion MRI reveals in vivo and non-invasively changes in astrocyte function induced by an aquaporin-4 inhibitor. PLoS One, 15(5), e0229702. https://doi.org/10.1371/journal.pone.0229702 
Den Heijer, T., der Lijn, F.v., Vernooij, M. W., de Groot, M., Koudstaal, P. J., der Lugt, A.v., ... Breteler, M. M. B. (2012). Structural and diffusion MRI measures of the hippocampus and memory performance. Neurolmage, 63(4), 1782-1789. https://doi.org/10.1016/j.neuroimage.2012.08.067

Elahy, M., Jackaman, C., Mamo, J. C., Lam, V., Dhaliwal, S. S., Giles, C., ... Takechi, R. (2015). Blood-brain barrier dysfunction developed during normal aging is associated with inflammation and loss of tight junctions but not with leukocyte recruitment. Immunity \& Ageing: I \& A, 12, 2. https://doi.org/10.1186/s12979-015-0029-9

Franco, C. Y., Petok, J. R., Langley, J., Hu, X., \& Bennett, I. J. (2020). Implicit associative learning relates to basal ganglia gray matter microstructure in young and older adults. Behavioural Brain Research, 397, 112950. https://doi.org/10.1016/j.bbr.2020.112950

Freitas, H. R., Ferreira, G. D. C., Trevenzoli, I. H., Oliveira, K. D. J., \& Reis, R. A. D. M. (2017). Fatty acids, antioxidants and physical activity in brain aging. Nutrients, 9(11), 1263. https://doi.org/10.3390/ nu9111263

Fukutomi, H., Glasser, M. F., Murata, K., Akasaka, T., Fujimoto, K., Yamamoto, T., ... Hayashi, T. (2019). Diffusion tensor model links to neurite orientation dispersion and density imaging at high b-value in cerebral cortical gray matter. Scientific Reports, 9(1), 12246. https:// doi.org/10.1038/s41598-019-48671-7

Fukutomi, H., Glasser, M. F., Zhang, H., Autio, J. A., Coalson, T. S., Okada, T., ... Hayashi, T. (2018). Neurite imaging reveals microstructural variations in human cerebral cortical gray matter. Neurolmage, 182, 488-499. https://doi.org/10.1016/J.NEUROIMAGE.2018.02.017

Ghadery, C., Pirpamer, L., Hofer, E., Langkammer, C., Petrovic, K., Loitfelder, M., ... Schmidt, R. (2015). R2* mapping for brain iron: Associations with cognition in normal aging. Neurobiology of Aging, 36(2), 925-932. https://doi.org/10.1016/j.neurobiolaging.2014.09.013

Grussu, F., Schneider, T., Tur, C., Yates, R. L., Tachrount, M., lanuş, A., ... Gandini Wheeler-Kingshott, C. A. M. (2017). Neurite dispersion: A new marker of multiple sclerosis spinal cord pathology? Annals of Clinical and Translational Neurology, 4(9), 663-679. https://doi.org/10. 1002/acn3.445

Guerrero, J. M., Adluru, N., Bendlin, B. B., Goldsmith, H. H., Schaefer, S. M., Davidson, R. J., ... Alexander, A. L. (2019). Optimizing the intrinsic parallel diffusivity in NODDI: An extensive empirical evaluation. PLoS One, 14(9), e0217118. https://doi.org/10.1371/journal.pone.0217118

Hallgren, B., \& Sourander, P. (1958). The effect of age on the non-haemin iron in the human brain. Journal of Neurochemistry, 3(1), 41-51. https://doi.org/10.1111/j.1471-4159.1958.tb12607.x

Jack, C. R., Jr, Twomey, C. K., Zinsmeister, A. R., Sharbrough, F. W., Petersen, R. C., \& Cascino, G. D. (1989). Anterior temporal lobes and hippocampal formations: normative volumetric measurements from MR images in young adults. Radiology, 172(2), 549-554. https://doi. org/10.1148/radiology.172.2.2748838

Jenkinson, M., Beckmann, C. F., Behrens, T. E. J., Woolrich, M. W., \& Smith, S. M. (2012). FSL. Neurolmage, 62(2), 782-790. https://doi.org/ 10.1016/J.NEUROIMAGE.2011.09.015

Kaden, E., Kelm, N. D., Carson, R. P., Does, M. D., \& Alexander, D. C. (2016). Multi-compartment microscopic diffusion imaging. Neurolmage, 139, 346-359. https://doi.org/10.1016/j.neuroimage. 2016.06.002

Langkammer, C., Krebs, N., Goessler, W., Scheurer, E., Ebner, F., Yen, K., ... Ropele, S. (2010). Quantitative MR imaging of brain iron: A postmortem validation study. Radiology, 257(2), 455-462. https://doi.org/10. 1148/radiol.10100495

Langley, J., Hussain, S., Flores, J. J., Bennett, I. J., \& Hu, X. (2020). Characterization of age-related microstructural changes in locus coeruleus and substantia nigra pars compacta. Neurobiology of Aging, 87(2), 8997. https://doi.org/10.1016/J.NEUROBIOLAGING.2019.11.016

Lezak, M. D. (1995). Neuropsychological assessment (3rd ed.). New York: Oxford University Press.
Lindenberger, U., von Oertzen, T., Ghisletta, P., \& Hertzog, C. (2011). Cross-sectional age variance extraction: What's change got to do with it? Psychology and Aging, 26(1), 34-47. https://doi.org/10.1037/ a0020525

Lister, J. P., \& Barnes, C. A. (2009). Neurobiological changes in the hippocampus during normative aging. Archives of Neurology, 66(7), 829-833. https://doi.org/10.1001/archneurol.2009.125

Macco, R., Pelizzoni, I., Consonni, A., Vitali, I., Giacalone, G., Martinelli Boneschi, F., ... Zacchetti, D. (2013). Astrocytes acquire resistance to iron-dependent oxidative stress upon proinflammatory activation. Journal of Neuroinflammation, 10(1), 897. https://doi.org/10.1186/ 1742-2094-10-130

Mackenzie, E. L., Iwasaki, K., \& Tsuji, Y. (2008). Intracellular iron transport and storage: From molecular mechanisms to health implications. Antioxidants and Redox Signaling, 10, 997-1030. https://doi.org/10.1089/ ars.2007.1893

Messinis, L., Nasios, G., Mougias, A., Politis, A., Zampakis, P., Tsiamaki, E., ... Papathanasopoulos, P. (2015). Age and education adjusted normative data and discriminative validity for Rey's auditory verbal learning test in the elderly greek population. Journal of Clinical and Experimental Neuropsychology, 38(1), 23-39. https://doi.org/10.1080/13803395. 2015.1085496

Metzler-Baddeley, C., Mole, J. P., Sims, R., Fasano, F., Evans, J., Jones, D. K., ... Baddeley, R. J. (2019). Fornix white matter glia damage causes hippocampal gray matter damage during age-dependent limbic decline. Scientific Reports, 9(1), 1060. https://doi.org/10.1038/ s41598-018-37658-5

Mills, E., Dong, X. P., Wang, F., \& Xu, H. (2010). Mechanisms of brain iron transport: Insight into neurodegeneration and CNS disorders. Future Medicinal Chemistry, 2, 51-64. https://doi.org/10.4155/fmc.09.140

Nasreddine, Z. S., Phillips, N. A., Bédirian, V., Charbonneau, S., Whitehead, V., Collin, I., ... Chertkow, H. (2005). The Montreal cognitive assessment, MoCA: A brief screening tool for mild cognitive impairment. Journal of the American Geriatrics Society, 53(4), 695-699. https://doi.org/10.1111/ j.1532-5415.2005.53221.x

Nazeri, A., Chakravart, M., Rotenberg, D. J., Rajji, T. K., Rathi, X., Michailovich, O. V., \& Voineskos, A. N. (2015). Functional consequences of neurite orientation dispersion and density in humans across the adult lifespan. Journal of Neuroscience, 35(4), 1753-1762. https://doi.org/10.1523/JNEUROSCI.3979-14.2015

Norenberg, M. D. (1994). Astrocyte responses to CNS injury. Journal of Neuropathology and Experimental Neurology, 53(3), 213-220. https:// doi.org/10.1097/00005072-199405000-00001

Patenaude, B., Smith, S. M., Kennedy, D. N., \& Jenkinson, M. (2011). A bayesian model of shape and appearance for subcortical brain segmentation. Neurolmage, 56(3), 907-922. https://doi.org/10.1016/j. neuroimage.2011.02.046

Pekny, M., \& Nilsson, M. (2005). Astrocyte activation and reactive gliosis. Glia, 50(4), 427-434. https://doi.org/10.1002/glia.20207

Pelizzoni, I., Zacchetti, D., Campanella, A., Grohovaz, F., \& Codazzi, F. (2013). Iron uptake in quiescent and inflammation-activated astrocytes: A potentially neuroprotective control of iron burden. Biochimica et Biophysica Acta - Molecular Basis of Disease, 1832(8), 1326-1333. https://doi.org/10.1016/j.bbadis.2013.04.007

Pendlebury, S. T., Welch, S. J. V., Cuthbertson, F. C., Mariz, J., Mehta, Z., \& Rothwell, P. M. (2017). Telephone assessment of cognition after TIA and stroke: TICSm and telephone MoCA vs face-to-face MoCA and neuropsychological battery. Stroke, 44(1), 227-229. https://doi.org/ 10.1161/STROKEAHA.112.673384.Telephone

Rae, C. L., Davies, G., Garfinkel, S. N., Gabel, M. C., Dowell, N. G., Cercignani, M., ... Critchley, H. D. (2017). Archival report deficits in neurite density underlie white matter structure abnormalities in first-episode psychosis. Biological Psychiatry, 82, 716-725. https://doi.org/10. 1016/j.biopsych.2017.02.008 
Radhakrishnan, H., Stark, S. M., \& Stark, C. E. L. (2020). Microstructural alterations in hippocampal subfields mediate age-related memory decline in humans. Frontiers in Aging Neuroscience, 12, 94. https://doi. org/10.3389/fnagi.2020.00094

Raz, N., \& Daugherty, A. M. (2018). Pathways to brain aging and their modifiers: Free-radical-induced energetic and neural decline in senescence (FRIENDS) model-a mini-review. Gerontology, 64, 49-57. https://doi.org/10.1159/000479508

Rey, A. (1941). L'examen psychologique dans les cas d'encéphalopathie traumatique. https://doi.org/1943-03814-001.

Rodrigue, K. M., Daugherty, A. M., Haacke, E. M., \& Raz, N. (2013). The role of hippocampal iron concentration and hippocampal volume in age-related differences in memory. Cerebral Cortex, 23(7), 1533-1541. https://doi.org/10.1093/cercor/bhs139

Schröder, N., Figueiredo, L. S., \& De Lima, M. N. M. (2013). Role of brain iron accumulation in cognitive dysfunction: Evidence from animal models and human studies. Journal of Alzheimer's Disease, 34(4), 797812. https://doi.org/10.3233/JAD-121996

Simon, M. J., \& Iliff, J. J. (2016). Regulation of cerebrospinal fluid (CSF) flow in neurodegenerative, neurovascular and neuroinflammatory disease. Biochimica et Biophysica Acta - Molecular Basis of Disease, 1862, 442-451. https://doi.org/10.1016/j.bbadis.2015.10.014

Singh, K., Trivedi, R., Devi, M. M., Tripathi, R. P., \& Khushu, S. (2016). Longitudinal changes in the DTI measures, anti-GFAP expression and levels of serum inflammatory cytokines following mild traumatic brain injury. Experimental Neurology, 275, 427-435. https://doi.org/10. 1016/j.expneurol.2015.07.016

Sofroniew, M. V. (2015). Astrogliosis. Cold Spring Harbor Perspectives in Biology, 7(2), a020420. https://doi.org/10.1101/cshperspect.a020420

Thomsen, M. S., Andersen, M. V., Christoffersen, P. R., Jensen, M. D., Lichota, J., \& Moos, T. (2015). Neurodegeneration with inflammation is accompanied by accumulation of iron and ferritin in microglia and neurons. Neurobiology of Disease, $81,108-118$. https://doi.org/10.1016/j. nbd.2015.03.013

Venkatesh, A., Stark, S. M., Stark, C. E. L., \& Bennett, I. J. (2020). Age- and memory- related differences in hippocampal gray matter integrity are better captured by NODDI compared to single-tensor diffusion imaging. Neurobiology of Aging, 96, 12-21. https://doi.org/10.1016/j. neurobiolaging.2020.08.004

Vilhardt, F., Haslund-Vinding, J., Jaquet, V., \& McBean, G. (2017). Microglia antioxidant systems and redox signalling. British Journal of Pharmacology, 174, 1719-1732. https://doi.org/10.1111/bph. 13426

Weber, M., Wu, T., Hanson, J. E., Alam, N. M., Solanoy, H., Ngu, H., ... Levie, K. S. (2015). Cognitive deficits, changes in synaptic function, and brain pathology in a mouse model of normal aging. ENeuro, 2(5), 47-62. https://doi.org/10.1523/ENEURO.0047-15.2015

Weber, R. A., Chan, C. H., Nie, X., Maggioncalda, E., Valiulis, G., Lauer, A., ... Adkins, D. A. L. (2017). Sensitivity of diffusion MRI to perilesional reactive astrogliosis in focal ischemia. NMR in Biomedicine, 30(7), e3717. https://doi.org/10.1002/nbm.3717

Yi, S. Y., Barnett, B. R., Torres-Velázquez, M., Zhang, Y., Hurley, S. A., Rowley, P. A., ... Yu, J. P. J. (2019). Detecting microglial density with quantitative multi-compartment diffusion MRI. Frontiers in Neuroscience, 13, 81. https://doi.org/10.3389/fnins.2019.00081

You, L. H., Yan, C. Z., Zheng, B. J., Ci, Y. Z., Chang, S. Y., Yu, P., ... Chang, Y. Z. (2017). Astrocyte hepcidin is a key factor in LPS-induced neuronal apoptosis. Cell Death \& Disease, 8(3), e2676. https://doi.org/ 10.1038/cddis.2017.93

Zecca, L., Youdim, M. B. H., Riederer, P., Connor, J. R., \& Crichton, R. R. (2004). Iron, brain ageing and neurodegenerative disorders. Nature Reviews Neuroscience, 5, 863-873. https://doi.org/10.1038/ nrn1537

Zhang, H., Schneider, T., Wheeler-Kingshott, C. A., \& Alexander, D. C. (2012). NODDI: Practical in vivo neurite orientation dispersion and density imaging of the human brain. Neurolmage, 61(4), 1000-1016. https://doi.org/10.1016/j.neuroimage.2012.03.072

Zhuo, J., Xu, S., Proctor, J. L., Mullins, R. J., Simon, J. Z., Fiskum, G., \& Gullapalli, R. P. (2012). Diffusion kurtosis as an in vivo imaging marker for reactive astrogliosis in traumatic brain injury. Neurolmage, 59(1), 467-477. https://doi.org/10.1016/j.neuroimage. 2011.07.050

How to cite this article: Venkatesh, A., Daugherty, A. M., \& Bennett, I. J. (2021). Neuroimaging measures of iron and gliosis explain memory performance in aging. Human Brain Mapping, 42(17), 5761-5770. https://doi.org/10.1002/hbm. 25652 